\title{
Preparation of tertiary amides from carbamoyl chlorides
}

\author{
and organocuprates
}

Laurent Lemoucheux, Thomas Seitz, Jacques Rouden, and Marie-Claire Lasne

Laboratoire de Chimie Moléculaire et Thio-organique, CNRS UMR 6507, ENSICaen, Université de

Caen-Basse Normandie, 6 Boulevard du Maréchal Juin, F14050 Caen Cedex, France

rouden@ismra.fr

\section{Supporting Information}

\section{General experimental details.}

Tetrahydrofuran (THF) was dried and distilled from sodium benzophenone ketyl. Ether was distilled from $\mathrm{LiAlH}_{4}$ and toluene from sodium. $\mathrm{CH}_{2} \mathrm{Cl}_{2}, \mathrm{Et}_{3} \mathrm{~N}$ and carbamoyl chlorides were distilled from $\mathrm{CaH}_{2}$ before use. Organolithium and Grignard reagent solutions were purchased and titrated before use. ${ }^{1} \mathrm{GC}$ analyses were performed on a 5\% Phenyl methylpolysiloxane column BPX5 (0.32 mm Internal Diameter, $30 \mathrm{~m}$ Length, 1 vm Film thickness), with 10 psi of nitrogen as gas carrier.

For Table 1: Amides 4a-e, 8a-e, 12a-e, formamides 5, 9, 13, ureas 6, 10, 14 and oxalamides 7, 11, 15 which were not commercially available, were synthesized by conventional methods using amines and acids or their derivatives. All purified compounds were analysed by GC: $120^{\circ} \mathrm{C}$ for $2 \mathrm{~min}, 120{ }^{\circ} \mathrm{C}$ to $250{ }^{\circ} \mathrm{C}$ with $20{ }^{\circ} \mathrm{C}$ per min, $250{ }^{\circ} \mathrm{C}$ for $6 \mathrm{~min} 30 \mathrm{sec}$. Calibration curves were established for each product for GC quantification.

GC-MS analyses were performed on a CP-Sil 8CB Low BLEED column (L: $30 \mathrm{~m}$, D: $0.25 \mathrm{~mm}$ ) and Electronic Impact was the method used on the MS detector.

\footnotetext{
${ }^{1}$ For organolithium solutions: Juaristi, E.; Martinez-Richa, A.; Garcia-Rivera, A.; Cruz-Sanchez, J. S. J. Org. Chem. 1983, 48, 2603-2606. For Grignard reagent solutions: Watson, S. C.; Eastham, J. F. J. Organomet. Chem. 1967, 9, 165-168.
} 


\section{General procedure of reactions using cyano-Gilman lithiocuprates (Table 1).}

Under a nitrogen atmosphere, the organolithium solution ( $7.2 \mathrm{mmol}, 2.4$ equiv) was slowly added to a cold $\left(-30{ }^{\circ} \mathrm{C}\right)$ suspension of $\mathrm{CuCN}\left(0.32 \mathrm{~g}, 3.6 \mathrm{mmol}, 1.2\right.$ equiv) in THF or $\mathrm{Et}_{2} \mathrm{O}(30 \mathrm{~mL})$. After 30 min, carbamoyl chloride $\left(3.0 \mathrm{mmol}, 1\right.$ equiv) was added at $-30{ }^{\circ} \mathrm{C}$. The reaction was stirred $2 \mathrm{~h}$ at cold temperature or $\mathrm{rt}$ (see table 1), quenched with a saturated $\mathrm{NH}_{4} \mathrm{Cl}$ solution $(2 \mathrm{~mL})$ and dried $\left(\mathrm{MgSO}_{4}\right)$. The volume was completed to $100 \mathrm{~mL}$ by addition of $\mathrm{CH}_{2} \mathrm{Cl}_{2}$ and a sample was injected to $\mathrm{GC}$ (quantification of products). After addition of a $28 \% \mathrm{NH}_{4} \mathrm{OH}$ solution $(30 \mathrm{~mL})$, stirring was pursued until the color of the solution turned deep blue. The reaction mixture was extracted with $\mathrm{CH}_{2} \mathrm{Cl}_{2}$, the combined organic layers were dried $\left(\mathrm{MgSO}_{4}\right)$ and concentrated to yield crude amides 4,8 and 12 which were purified (by flash chromatography or Kugelrohr distillation) when necessary.

\section{General procedure of reactions using cyano-Gilman magnesiocuprates (Table 2).}

Under a nitrogen atmosphere, the organomagnesium solution (13.66 mmol, 2.1 equiv) was slowly added to a cold $\left(-30{ }^{\circ} \mathrm{C}\right)$ suspension of $\mathrm{CuCN}\left(0.61 \mathrm{~g}, 6.82 \mathrm{mmol}, 1.05\right.$ equiv) in $\mathrm{Et}_{2} \mathrm{O}(60 \mathrm{~mL})$. After $45 \mathrm{~min}$, carbamoyl chloride $\left(6.50 \mathrm{mmol}, 1\right.$ equiv) was added at $-30{ }^{\circ} \mathrm{C}$, the reaction mixture was slowly warmed to rt and stirred $15 \mathrm{~h}$. The reaction were quenched with a saturated $\mathrm{NH}_{4} \mathrm{Cl}$ solution $(10 \mathrm{~mL})$ and after addition of a $28 \% \mathrm{NH}_{4} \mathrm{OH}$ solution $(20 \mathrm{~mL})$, stirring was pursued until the color of the solution turned deep blue. The reaction mixture was extracted with $\mathrm{CH}_{2} \mathrm{Cl}_{2}$, the combined organic layers were dried $\left(\mathrm{MgSO}_{4}\right)$ and concentrated to yield crude amides $\mathbf{8}$ which were purified (by flash chromatography or Kugelrohr distillation) when necessary. 
Table 1. Qualitative and quantitative results of the reaction between carbamoyl chlorides and cyanoGilman reagents

\begin{tabular}{|c|c|c|c|c|c|c|}
\hline$R^{3}$ & & $\stackrel{\left(\mathrm{R}^{3}\right)_{2} \mathrm{CuLi} \text {.LiCN }}{\longrightarrow}$ & $\mathrm{O}$ & $\mathrm{O}$ & $\mathrm{O}$ & 요쓴 \\
\hline $\begin{array}{l}\text { a n-Bu } \\
\text { b s-Bu }\end{array}$ & $1 \mathrm{X}=\mathrm{NEt}_{2}$ & IHF or $\mathrm{Et}_{2} \mathrm{O}$ & $4 a-e$ & 5 & 6 & 7 \\
\hline $\begin{array}{l}\text { ct-Bu } \\
\text { d Ph }\end{array}$ & $2 x=\sqrt{ } \mathrm{O}$ & & $8 a-e$ & 9 & 10 & 11 \\
\hline e Me & $3 x=$ & & $12 a-e$ & 13 & 14 & 15 \\
\hline
\end{tabular}

\begin{tabular}{|c|c|c|c|c|c|c|c|c|c|c|c|c|c|c|c|c|}
\hline \multirow[t]{3}{*}{ entry } & \multirow[t]{3}{*}{$\mathrm{sm}^{\mathrm{a}}$} & \multirow{3}{*}{$\begin{array}{c}\text { cuprate }^{\mathrm{b}} \\
\mathrm{R}^{3}\end{array}$} & \multicolumn{2}{|c|}{$\mathrm{T}\left({ }^{\circ} \mathrm{C}\right)$} & \multicolumn{3}{|c|}{ amide $(\%)^{\mathrm{c}}$} & \multicolumn{3}{|c|}{ formamide $(\%)^{\mathrm{c}}$} & \multicolumn{3}{|c|}{ urea $(\%)^{\mathrm{c}}$} & \multicolumn{3}{|c|}{ oxalamide $(\%)^{\mathrm{c}}$} \\
\hline & & & THF & $\mathrm{Et}_{2} \mathrm{O}$ & & THF & $\mathrm{Et}_{2}$ & & THF & $\mathrm{Et}_{2}$ & & THF & $\mathrm{Et}_{2}$ & & THF & $\mathrm{Et}_{2}$ \\
\hline & & & & & & & $\mathrm{O}$ & & & $\mathrm{O}$ & & & $\mathrm{O}$ & & & $\mathrm{O}$ \\
\hline 1 & 1 & $n-\mathrm{Bu}$ & -30 to 0 & -30 to $\mathrm{rt}$ & $4 a$ & 85 & 70 & 5 & 0 & 2 & 6 & 5 & 0 & 7 & 0 & 0 \\
\hline 2 & 1 & $s-\mathrm{Bu}$ & -30 to 0 & -30 to 0 & $4 b$ & 90 & 62 & 5 & 0 & 11 & 6 & 0 & 0 & 7 & 0 & 0 \\
\hline 3 & 1 & $t$-Bu & -30 to 0 & -30 to 0 & $4 c$ & $37^{\mathrm{f}}$ & $33^{\mathrm{f}}$ & 5 & 0 & 8 & 6 & 4 & 13 & 7 & 0 & 3 \\
\hline 4 & 1 & $\mathrm{Ph}$ & -30 to $\mathrm{rt}$ & -30 to $\mathrm{rt}$ & $4 d$ & 50 & 70 & 5 & 0 & 0 & 6 & 2 & 16 & 7 & 0 & 0 \\
\hline 5 & 1 & $\mathrm{Me}$ & -30 to rt & -30 to 0 & $4 e$ & 90 & 80 & 5 & 0 & 0 & 6 & 0 & 0 & 7 & 0 & 0 \\
\hline 6 & 2 & $n$-Bu & -30 & -30 to 0 & $8 a$ & $83^{e}$ & 38 & 9 & 0 & 2 & 10 & 9 & 25 & 11 & 4 & 0 \\
\hline 7 & 2 & $s-\mathrm{Bu}$ & -30 to $\mathrm{rt}$ & -30 to $\mathrm{rt}$ & $\mathbf{8 b}$ & $50^{\mathrm{e}}$ & $33^{\mathrm{d}}$ & 9 & 2 & 8 & 10 & 8 & 4 & 11 & 14 & 4 \\
\hline 8 & 2 & $t$-Bu & -30 & -30 to $\mathrm{rt}$ & $8 c$ & $62^{\mathrm{e}}$ & $43^{\mathrm{d}}$ & 9 & 2 & 5 & 10 & 9 & 6 & 11 & 1 & 4 \\
\hline 9 & 2 & $\mathrm{Ph}$ & -30 & -30 to 0 & $8 d$ & 64 & 70 & 9 & 0 & 0 & 10 & 4 & 4 & 11 & 4 & 3 \\
\hline 10 & 2 & $\mathrm{Me}$ & -30 & -30 to $\mathrm{rt}$ & $8 \mathrm{e}$ & 30 & 20 & 9 & 0 & 0 & 10 & 2 & 16 & 11 & 8 & 4 \\
\hline 11 & 3 & $n-\mathrm{Bu}$ & -30 & -30 to 0 & $12 \mathrm{a}$ & 75 & 70 & 13 & 0 & 4 & 14 & 6 & 5 & 15 & 0 & 0 \\
\hline 12 & 3 & $s-\mathrm{Bu}$ & -30 to $\mathrm{rt}$ & -30 to $\mathrm{rt}$ & $12 b$ & 75 & 70 & 13 & 0 & 10 & 14 & 1 & 1 & 15 & 1 & 0 \\
\hline 13 & 3 & $t$-Bu & -30 to 0 & -30 to 0 & $12 \mathrm{c}$ & $85^{\mathrm{e}}$ & $55^{\mathrm{f}}$ & 13 & 0 & 1 & 14 & 0 & 1 & 15 & 0 & 0 \\
\hline 14 & 3 & $\mathrm{Ph}$ & -30 to 0 & -30 to 0 & 12d & 84 & 83 & 13 & 0 & 0 & 14 & 5 & 5 & 15 & 1 & 0 \\
\hline 15 & 3 & $\mathrm{Me}$ & -30 & -30 & $12 \mathrm{e}$ & 70 & 68 & 13 & 0 & 0 & 14 & 1 & 0 & 15 & 0 & 0 \\
\hline
\end{tabular}

${ }^{\mathrm{a}}$ Starting material. ${ }^{\mathrm{b}}$ Cyanocuprate reagents were prepared at $-30{ }^{\circ} \mathrm{C}$. Reaction time: $2 \mathrm{~h} .{ }^{\mathrm{c}}$ GC yields. d $5-10 \%$ unreacted carbamoyl chloride. ${ }^{\text {e }} 10-20 \%$ unreacted carbamoyl chloride. ${ }^{\mathrm{f}}$ 20-40\% unreacted carbamoyl chloride. 